\title{
Eficacia y seguridad de la terapia tópica con capsaicina $0,075 \%$ versus mentol $1 \%$, en el tratamiento del prurito de la foliculitis eosinofílica asociada al virus de la inmunodeficiencia adquirida
}

\author{
Carlos Galarza ${ }^{1,2,3}$, Willy Ramos ${ }^{1,3}$, Humberto Chía ${ }^{3}$, Gerardo Ronceros ${ }^{1}$, \\ Jesús Santiani ${ }^{1}$, Ericson Gutiérrez ${ }^{1}$, Marcos Ñavincopa ${ }^{4,5}$, María Vilcarromero ${ }^{1}$, \\ Jack Ávila ${ }^{1}$, Jorge Hancco ${ }^{1}$, Deny Gámez ${ }^{6}$
}

Resumen

Objetivo: Determinar la eficacia y seguridad de la terapia tópica con capsaicina 0,075\% versus mentol $1 \%$ en el tratamiento del prurito de la foliculitis eosinofilica asociada al virus de la inmunodeficiencia adquirida (VIH). Diseño: Estudio clínico aleatorizado y doble ciego. Lugar: Servicios de Enfermedades Infecciosas y Tropicales (SEIT) y Dermatología, Hospital Nacional Dos de Mayo, Lima, Perú. Participantes: Pacientes con foliculitis eosinofilica asociada al VIH. Intervenciones: La muestra estuvo constituida por 40 pacientes con foliculitis eosinofilica asociada al VIH, quienes fueron distribuidos mediante muestreo aleatorio sistemático en dos grupos: el grupo 1 conformado por 20 pacientes que recibieron la terapia 1 ; y el grupo 2 , conformado por 20 pacientes que recibieron la terapia 2. Los pacientes recibieron tratamiento con capsaicina al 0,075\% y mentol 1\%; la aplicación se realizó en el área afectada, cada 6 horas, durante 45 días. Se implementó un sistema de visitas (domiciliarias u hospitalarias), se evaluó la eficacia en la reducción del prurito (escala de Likert) y se registró los eventos. Principales medidas de resultados: Respuesta clínica a la administración de capsaicina y mentol. Resultados: La respuesta fue buena en $90 \%$ de los pacientes que recibieron capsaicina $0,075 \%$, en comparación con $40 \%$ en el grupo que recibió mentol al $1 \%(p=0,001)$, con OR $=0,074$ (IC $95 \%: 0,013-0,411)$. La eficacia de las terapias tópicas fue modificada por el recuento de linfocitos CD4 ( $p<0,001)$. La presencia de enfermedades oportunistas $(p=0,252)$ no afectó la eficacia de las terapias. Se presentaron efectos adversos locales (eritema y ardor) en 3 pacientes (15\%) que recibieron capsaicina $0,075 \%$, mientras que en el grupo que recibió mentol al $1 \%$ un paciente (5\%) presentó eritema, no hallándose diferencia estadísticamente significativa $(p>0,05)$. Conclusiones: La terapia tópica con capsaicina es eficaz y segura en el tratamiento del prurito, en la foliculitis eosinofilica asociada al VIH.

Palabras clave Foliculitis; VIH; capsaicina; prurito; mentol.

Topical capsaicin $0,075 \%$ versus menthol $1 \%$ efficacy and safety in HIV-associated eosinofilic folliculitis

Abstract

Objetive: To determine efficacy and safety of capsaicin $0,075 \%$ topical therapy in the treatment of pruritus due

1 Instituto de Investigaciones Clínicas, Facultad de Medicina Universidad Nacional Mayor de San Marcos. Lima, Perú.

2 Servicio de Dermatología, Hospital Nacional Dos de Mayo. Lima, Perú.

3 Servicio Académico Asistencial de Dermatología, Facultad de Medicina - Universidad Nacional Mayor de San Marcos. Lima, Perú

4 Instituto de Medicina Tropical Daniel Alcides Carrión, Facultad de Medicina - Universidad Nacional Mayor de San Marcos. Lima, Perú.

5 Servicio de Enfermedades Infecciosas y Tropicales, Hospital Nacional Dos de Mayo. Lima, Perú.

6 Clínica San Pablo. Lima, Perú. to eosinophilic folliculitis in HIV patients with lymphocyte count CD4 (+) $<250 \times \mathrm{mm}^{3}$. Design: Randomized clinical trial, double blind study. Setting: Infectious and Tropical Diseases Service and Dermatology Service, Hospital Nacional Dos de Mayo, Lima, Peru. Participants: Patients with VIH-associated eosinophilic folliculitis. Interventions: Forty patients with HIV-associated eosinophilic folliculitis were randomly distributed in two groups: group 1 included 20 patients that received sample 1 and group 2, 20 patients that received sample 2. Patients received capsaicin 0,075\% and menthol 1\% treatments (marked as sample 1 and sample 2). Instructions on topical use each 6 hours were given to patients. Visits were programmed to evaluate efficacy and monitor adverse events by study drugs. Main outcome measures: Capsaicin and menthol administration 
clinical outcome. Results: We found good response in $90 \%$ of patients receiving capsaicin 0,075\% (18/20) versus $40 \%$ of the group treated with menthol $1 \%(88 / 20)$, with significant statistical difference $(p=0,001), O R=0,074 \%$ (IC 95\%: 0,013 - 0,411). Topical therapy efficacy was altered by CD4 lymphocyte count ( $<<0,001)$; otherwise the occurrence of opportunistic diseases $(p=0,252)$ did not affect therapy efficacy. There were local adverse events (erithema and heat) in 3 patients (15\%) who received capsaicin 0,075\% and only in one patient (5\%) of group 2 (menthol) who presented erithema (5\%), with no significant statistical difference $(p>0,05)$. Conclusions: Capsaicin topical therapy is effective and safe in the treatment of pruritus in HIV-associated eosinophilic folliculitis.

Key words: Folliculitis; HIV; capsaicin; pruritus; menthol.

\section{INTRODUCCIÓN}

Con la implementación de la terapia antirretroviral de gran actividad (TARGA) para pacientes con infección por VIH-sida, ha disminuido la frecuencia de infecciones graves en este grupo de pacientes, lo cual ha incrementado la longevidad de los pacientes y con esto la frecuencia de enfermedades crónicas de la piel $\left({ }^{1}\right)$.

El espectro de las enfermedades de la piel en pacientes con infección VIH incluye múltiples etiologías, muchas de las cuales pueden causar prurito intratable, que ocasiona lesiones por rascado que llegan a ser desfigurantes, causar pérdida del sueño y significativo estrés psicológico. Las causas del prurito incluyen infecciones cutáneas, infestaciones, desórdenes papuloescamosos, fotodermatitis, xerosis, erupciones farmacológicas y ocasionalmente desórdenes linfoproliferativos. Dentro de estos desórdenes se incluye a la foliculitis eosinofílica $\left({ }^{1}\right)$.

La foliculitis eosinofílica fue descrita originalmente por Ofuji y col. (1970); sin embargo, su asociación con el VIH fue reconocida recién en 1986, con algunas diferencias con la descripción original en su distribución, aparición de las lesiones, curso y síntomas de la enfermedad. El síntoma más característico es el prurito asociado a la aparición de una erupción papular, que compromete la cara, cuello, región superior del tórax, espalda y región proximal de los brazos. La patogenia es desconocida, pero se ha sugerido que la enfermedad podría ser resultado de una disregulación del sistema inmune a una variedad de agentes, como Pityrosporum ovale, Demodex foliculorum, o una reacción autoinmune al sebo o algún componente de este $\left({ }^{1,2}\right)$.

El tratamiento de la foliculitis eosinofílica es difícil, en función de que el mecanismo subyacente de la enfermedad es desconocido. Las variedades de tratamiento incluyen esteroides tópicos, acaricidas tópicos, fototerapia (luz ultravioleta A y B), itraconazol, isotretinoína y metronidazol. Todos estos fármacos han sido exitosos transitoriamente, pero las series estudiadas incluyen un pequeño número de pacientes y tienden más a ser comunicaciones anecdóticas que ensayos controlados o aleatorios. La enfermedad es recidivante y luego de la suspensión del tratamiento reaparece el prurito. Por este motivo, no existe consenso sobre cuál de los tratamientos debe ser indicado $\left(^{2-4}\right)$.

La capsaicina es un derivado del ají (Capsicum $s p$ ), conocido y utilizado desde el período precerámico en nuestro país, en la alimentación (condimento), con fines rituales, medicinales y de tortura $\left(^{5-15}\right)$. La capsaicina ha sido utilizada para el tratamiento tópico de enfermedades dolorosas que producen prurito e inflamación. Sus efectos consisten en excitación selectiva y posterior desensibilización de las fibras nerviosas nociceptivas de tipo $\mathrm{C}$, con secreción y posterior reducción de la sustancia $\mathrm{P}$. Estudios realizados en la terapia tópica de la neuralgia posherpética han mostrado que su uso es eficaz y seguro (16$\left.{ }^{25}\right)$. Asimismo, Lysy y col. (2003) comunican el uso de la capsaicina tópica para el tratamiento del prurito anal idiopático intratable, en un estudio aleatorio y controlado con placebo; de este modo, la capsaicina $0,006 \%$ fue más eficaz que el mentol $1 \%$ y el placebo $\left({ }^{26}\right)$.

Por la naturaleza de la terapia tópica con capsaicina y su eficacia en el tratamiento del prurito, se plantea el presente estudio, que busca determinar la eficacia de la capsaicina tópica $0,075 \%$ en el tratamiento del prurito en la foliculitis eosinofílica y compararla con el mentol $1 \%$. 


\section{MÉTODOS}

Estudio clínico aleatorizado y doble ciego. La muestra del estudio estuvo constituida por 40 pacientes que acudieron al consultorio externo de los Servicios de Enfermedades Infecciosas y Tropicales (SEIT) y Dermatología, así como pacientes hospitalizados en el SEIT del Hospital Nacional Dos de Mayo, Lima, Perú, a quienes se les diagnosticó foliculitis eosinofílica asociada al VIH y que cumplieron con los criterios de inclusión y exclusión. Los criterios de inclusión fueron diagnóstico de foliculitis eosinofílica, estudio anatomopatológico confirmatorio, prurito, edad entre los 18 y 45 años, firma del consentimiento informado y tener recuento CD $4<250 \mathrm{x} \mathrm{mm}^{3}$. Se excluyó a los pacientes que presentaron al momento del estudio otra patología dermatológica causante de prurito o tratamiento tópico dos semanas antes del ingreso al estudio.

Se consideró a 40 pacientes, que fueron distribuidos mediante muestreo aleatorio sistemático en dos grupos: el grupo 1 conformado por 20 pacientes, que recibieron la muestra 1 , si su historia clínica terminaba con número impar $(1,3,5 \ldots \ldots)$, y el grupo 2 , conformado por 20 pacientes que recibieron la muestra 2 , si su historia clínica terminaba con número par $(2,4,6 \ldots .$.$) . Los pacientes$ recibieron tratamiento con capsaicina al $0,075 \%$ y mentol $1 \%$, los cuales fueron rotulados como muestra 1 o muestra 2 . Ni el investigador ni el investigado tuvieron conocimiento de la sustancia que correspondía a la muestra 1 o 2 .

A los 40 pacientes que ingresaron al estudio se les entregó un pote del fármaco en estudio. La cantidad entregada fue de 45 gramos, cálculo establecido para utilizar 1 gramo por día. Se les indicó el modo de aplicación, el cual se realizó mediante la administración de una capa fina en el área afectada, usando un hisopo, cada 6 horas, luego de lavarse las manos y conservar el pote refrigerado. Se implementó un sistema de visitas (domiciliarias u hospitalarias), las cuales se realizó a los 15, 30 y 45 días, a cargo de un médico dermatólogo, con la finalidad de evaluar la eficacia y monitorear la presencia de eventos adversos relacionados a la medicación en estudio. La cuantificación de la intensidad del prurito se realizó mediante escala de Likert, siendo $0=$ ausencia de prurito, $1=$ prurito leve, 2 = prurito moderado, $3=$ severo, $4=$ extremo.

La eficacia se evaluó comparando la respuesta con cada fármaco, al inicio y a los 15,30 y 45 días. Se consideró como mejoría ( + ) cuando disminuyó el prurito 2 grados en la escala, de 4 a 2 , de 3 a 1 o de 2 a 0 . Se consideró mejoría (-) si disminuyó solo 1 grado en la escala, no mejoró o empeoró.

Para el análisis estadístico, se utilizó el programa estadístico SPSS versión 13,0; para la estadística bivariada se usó la prueba chi cuadrado y la prueba t de student. Para determinar la eficacia de las terapias tópicas con capsaicina y mentol, se empleó estadística multivariada, mediante regresión logística múltiple, con control de variables confusoras (recuento de linfocitos CD4+ y presencia de infecciones oportunistas), calculándose el odds ratio (OR). Los cálculos fueron realizados con un intervalo de confianza del $95 \%$.

Con relación a los aspectos éticos, los pacientes incluidos en el estudio, luego de haberles explicado los objetivos del mismo y de haber aceptado las condiciones, firmaron el consentimiento informado, proporcionándole una copia y quedando el original en el archivo del estudio.

\section{RESULTADOS}

La edad promedio de los pacientes de ambos grupos de investigación fue 35,8 $\pm 6,2$ años, $65 \%$ correspondió al sexo masculino y el $35 \%$ restante, al sexo femenino. El tiempo de enfermedad promedio fue $1,9 \pm 0,8$ años, para el grupo tratado con capsaicina, mientras que para el grupo tratado con mentol $1 \%$ fue $0,8 \pm 0,6$ años. Las características de ambos grupos puede observarse en la Tabla 1.

Al evaluarse la eficacia de ambas terapias tópicas, se encontró que la respuesta a los 45 días de terapia fue buena en $90 \%$ de los pacientes que recibieron capsaicina $0,075 \%(18 / 20)$, en comparación con $40 \%$ en el grupo que recibió mentol $1 \%(8 / 20)$, para lo cual existió diferencia estadísticamente significativa $(p=0,007)$, calculándose un $\mathrm{OR}=0,09$ 
Tabla 1. Características de los grupos de estudio.

\begin{tabular}{lccc}
\hline \multicolumn{1}{c}{ Característica } & Capsaicina & Mentol & Valor \\
& $0,075 \%$ & $1 \%$ & $p$ \\
\hline Superficie corporal afectada & $58,25 \%$ & $58,23 \%$ & $0,995^{*}$ \\
Infecciones oportunistas & $75 \%$ & $60 \%$ & $0,078^{\dagger}$ \\
TARGA & $65 \%$ & $40 \%$ & $0,102^{\dagger}$ \\
\hline
\end{tabular}

${ }^{\dagger}$ Prueba chi cuadrado.

* Prueba t de student, para muestras independientes.

(IC 95\%: 0,02-0,52). La eficacia de las terapias tópicas fue modificada por el recuento de linfocitos CD4 $(p<0,001)$; por otro lado, la presencia de enfermedades oportunistas $(p=0,252)$ no afectó la eficacia de las terapias (Tabla 2).

Se presentaron efectos adversos locales (eritema y ardor) en 3 pacientes (15\%) que recibieron capsaicina $0,075 \%$, mientras que en el grupo que recibió mentol al $1 \%$ un paciente $(5 \%)$ presentó eritema, no hallándose diferencia estadísticamente significativa $(p=0,302)$.

\section{DISCUSIÓN}

La foliculitis eosinofílica asociada al VIH es una entidad clínica de difícil manejo, con un impacto importante en la calidad de vida de los pacientes. El presente estudio muestra que la terapia tópica con capsaicina al $0,075 \%$ es eficaz y segura en el tratamiento de esta enfermedad $\left({ }^{1-4}\right)$; además, se observa que la disminución del prurito es estadísticamente

Tabla 2. Respuesta al tratamiento a los 15, 30 y 45 días del tratamiento tópico con capsaicina $0,075 \%$ y mentol $1 \%$, en pacientes con prurito de la foliculitis eosinofílica asociada al VIH.

\begin{tabular}{cccc}
\hline $\begin{array}{c}\text { Tratamiento } \\
\text { tópico }\end{array}$ & $\begin{array}{c}\text { Respuesta a } \\
\text { los 15 días }\end{array}$ & $\begin{array}{c}\text { Respuesta a } \\
\text { los 30 días }\end{array}$ & $\begin{array}{c}\text { Respuesta a } \\
\text { los 45 días }\end{array}$ \\
\hline Capsaicina 0,075\% & Buena: $20 \%$ & Buena: 65\% & Buena: $90 \%$ \\
Mala: $80 \%$ & Mala: $35 \%$ & Mala: $10 \%$ \\
Mentol 1\% & Buena: 5\% & Buena: $15 \%$ & Buena: $40 \%$ \\
& Mala: $95 \%$ & Mala: $85 \%$ & Mala: $60 \%$ \\
Valor $p^{*}$ & 0,171 & 0,002 & 0,001 \\
\hline
\end{tabular}

* Prueba chi cuadrado. significativa a partir de los 30 días, con una respuesta máxima a los 45 días de tratamiento tópico.

La capsaicina es un fármaco usado como terapia tópica del dolor neuropático, particularmente de la neuralgia posherpética. El efecto de la capsaicina en el tratamiento de la foliculitis eosinofílica se fundamenta en que tanto el dolor como el prurito se transmiten mediante fibras nerviosas amielínicas del tipo $\mathrm{C}$, a nivel del sistema nervioso y sus terminaciones en la epidermis.

Se sabe que uno de los principales neuropéptidos mediadores de la transmisión del dolor y del prurito desde el sistema nervioso periférico al sistema nervioso central, es la sustancia P. La capsaicina, al actuar sobre las neuronas aferentes de tipo $\mathrm{C}$ a través de sus receptores vaniloides, tiene un efecto inhibidor sobre la síntesis, transporte, almacenamiento y liberación de la sustancia P, lo cual explicaría su efecto sobre el prurito $\left({ }^{26}\right)$. Los mecanismos mediante los cuales la capsaicina reduce el prurito no son totalmente comprendidos y actualmente se discute sobre si es que las fibras para la transmisión del prurito y el dolor son las mismas $\left({ }^{26}\right)$.

No existen reportes previos del uso de capsaicina en foliculitis eosinofílica asociada al VIH. Sin embargo, la buena respuesta en el manejo del prurito anal idiopático intratable (Lysy, 2003) ${ }^{(26}$ ) y pitiriasis rubra pilaris (Neess, 2000) $\left({ }^{27}\right)$, así como nuestra experiencia $\left({ }^{24,25}\right)$ de la tolerabilidad del tratamiento por los pacientes y baja frecuencia de eventos adversos locales, hacen de la capsaicina una alternativa para el manejo tópico del prurito. Se escogió la concentración de capsaicina al 0,075\%, debido a que ésta había mostrado ser la dosis de mayor eficacia en el tratamiento del dolor en otros estudios.

La principal limitación de nuestro estudio es el tamaño de la muestra, que es de solo 20 pacientes, por grupo de investigación. Es por esta razón que consideramos necesaria la realización de estudios multicéntricos que incluyan un mayor número de pacientes, así como la concentración mínima necesaria de capsaicina para un efecto óptimo, con lo que se reduciría aún más la frecuencia de eventos adversos. 


\section{REFERENCIAS BIBLIOGRÁFICAS}

1. Singh F, Rudikoff D. VIH-Associated Pruritus. Etiology and Management. Am J Clin Dermatol. 2003;4:177-88.

2. Simpson-Dent SL, Fierfield LA, staughton RC. VIHassociated eosinophilic follicullitis- differential diagnosis and management. Sex Transm Inf. 1999;75:291-3.

3. Moyle M, Woolley IJ, Thevarajan I, Korman TM. Eosinophilic follicullitis: an example of immune reconstitution follicullitis. AIDS. 2004;18:2350-2.

4. Tang MBY, Tan E, Hon Chua S. Eosinophilic pustular follicullitis (Ofuji's disease) in singapore: A review of 23 adult cases. Australas J Dermatol. 2003;44:44-7.

5. Mechán V. Medicina Prehispánica [Tesis doctoral]. Lima: Facultad de Medicina, Universidad Peruana Cayetano Heredia; 2002.

6. Thomas LF, Kennet KAR. Early human cultural and skeletal remains. En: Lynch T (Ed). Guitarrero cave. Early man in the Andes. New York: Academic Press; 1980.

7. McNeish RS, Nelken-Terner A, Garcia A. First annual report of the Ayacucho Archaeeological Botanical Project. National Science Foundation- Robert S Peabody Fundation for Archeology. Andover, Massachusetts: Phillips Academy; 1969.

8. McNeish RS, Nelken-Terner A, Garcia A. Second annual report of the Ayacucho Archaeeological Botanical Project. National Science Foundation. Andover, Massachusetts. Robert S Peabody Fundation for Archeology / Phillips Academy; 1970.

9. Bonavia D. Coprolitos y dieta del precerámico tardío de la costa peruana. Bull Inst Fr Et And. 1985;14:85-140.

10. Bonavia D, Johnson L, Reitz E, Wing E, Weir G. Un sitio precerámico de Huarmey antes de la introducción del maíz. Bull Institut Fr Etudes Andines. 1993;22:409-42.

11. Bonavia D. Los camélidos sudamericanos. Una introducción a su estudio. Lima: IFEA/UPCH/Conservation Internacional; 1996.

12. Callen E, Cameron T. The diet and parasites of prehistoric Huaca Prieta indians as determined by dried coprolites. Proceedings Royal Society of Canada. 1955;5:51-2.

13. Callen EO. La dieta revelada por los coprolitos. En: Brothwell D (Compilador), Clark G (Compilador), Higos E (Compilador), Sánchez M (Traductor). Ciencia en Arqueología. México: Fondo de Cultura Económica, 1980. p. 240-8.

14. Cabieses F. Antropología del ají. Lima: Ministerio de Salud del Perú; 2000.

15. Antúnez de Mayolo SE. La nutrición en el antiguo Perú. Lima: Banco Central de Reserva del Perú; 1981.

16. Abeldaño A. Capsaicina, su uso en dermatología. Act Terap Dermatol. 1997;20:205-8.
17. Alper BS, Lewis PR. Treatment of postherpetic neuralgia: a systematic review of the literature. J Fam Pract. 2002;51:121-8.

18. Buck SH, Burks TF. The neuropharmacology of capsaicin: Review of some recent observations. Pharmacol Rev. 1986;38:179-226.

19. Bernstein JE, Bickers DR, Dahl MV, Roshal JY. Treatment of chronic postherpetic neuralgia with topical capsaicin. A preliminary study. J Am Acad Dermatol. 1987;17:93-6.

20. Watson CP, Evans RJ, Watt VR. Post herpetic neuralgia and topical capsaicin. Pain. 1988;33:333-40.

21. Bernstein JE, Korman NJ, Bickers DR, Dahl MV, Millikan LE. Topical capsaicin treatment of chronic postherpetic neuralgia. J Am Acad Dermatol. 1989;21:265-70.

22. Peikert A, Hentrich M, Ochs G. Topical 0,025\% capsaicin in chronic post-herpetic neuralgia: Efficacy, predictors of response and long-term course. J Neurol. 1991;238:452-6.

23. Watson CPN, Tyler KL, Bickers DL, Millikan LE, Smith S, Coleman E. A randomized vehicle-controlled trial of topical capsaicin in the treatment of postherpetic neuralgia. Clin Ther. 1993; 15:510-26.

24. Galarza C, Ortega A, Ramos W, Ávila J, Hurtado J, Lindo G. Eficacia y seguridad del tratamiento tópico con capsaicina $0,05 \%$ versus capsaicina $0,075 \%$ en la neuralgia postherpética. Ensayo clínico aleatorizado. Lima-Perú. 2003. Ann Fac Med. 2004;65:s33.

25. Galarza C. Eficacia y seguridad del tratamiento tópico con capsaicina $0,05 \%$ versus capsaicina $0,075 \%$ en la neuralgia postherpética. Hospital Nacional Dos de Mayo. Marzo 2003 - febrero 2004. Dermatol Perú. 2005;15:108-12.

26. Lysy J, Sistiery-Ittah M, Israelit Y, Shmueli A, StraussLiviatan N, Mindrul V, et al. Topical capsaicin-a novel and effective treatment for idiopathic intractable pruritus ani: a randomized, placebo controlled, crossover study. Gut. 2003;52:1323-6.

27. Neess CM, Hinrichs R, Dissemond J, Hermmann G, Poswig A, Servera-Llanras M, et al. Treatment of pruritus by capsaicin in a patient with pityriasis rubra pilaris receiving RE-PUVA therapy. Clin Dermatol. 2000;25:209-11.

Manuscrito recibido el 05 de setiembre de 2007 y aceptado para publicación el 26 de setiembre de 2007.

Correspondencia:

Dr. Carlos Galarza Manyari

Hospital Nacional Dos de Mayo

Instituto de Investigaciones Clínicas - UNMSM

Av. Grau, cuadra 13, Parque de la Medicina Peruana

Lima, 1 Perú

Correo-e: investcl@yahoo.com 